\title{
THE COMPACTNESS OF A WEAKLY SINGULAR INTEGRAL OPERATOR ON WEIGHTED SOBOLEV SPACES
}

\author{
DAVID ELLIOTT
}

\begin{abstract}
It is shown that the weakly singular integral operator $\int_{-1}^{1}\left(\phi(\tau) /|\tau-t|^{\gamma}\right) d \tau$, where $0<\gamma<1$, maps the weighted Sobolev space $W_{p ; \alpha, \beta}^{(n)}(\Omega)$ compactly into itself for $1<p<\infty, 0<\alpha+1 / q, \beta+1 / q<1$ and $n \in \mathbb{N}_{0}$.
\end{abstract}

1. Introduction. We shall start by defining the weighted Sobolev spaces of the title. Let $\mathbb{N}_{0}$ denote the set of all non-negative integers, so that $\mathbb{N}_{0}:=\{0,1,2, \ldots\}=:\{0\} \cup \mathbb{N}$, and let $\Omega$ denote the interval $(-1,1)$.

Definition 1.1. For $1 \leq p<\infty$, real $\alpha$ and $\beta$, and $n \in \mathbb{N}_{0}$, we shall denote by $W_{p ; \alpha, \beta}^{(n)}(\Omega)$ the space of all functions $\phi$ such that

$$
I_{j}(\phi):=\int_{-1}^{1}\left((1-\tau)^{j-\alpha}(1+\tau)^{j-\beta}\left|\phi^{(j)}(\tau)\right|\right)^{p} d \tau
$$

is finite, for all $j=0(1) n$. A norm on the space $W_{p ; \alpha, \beta}^{(n)}(\Omega)$ will be denoted and defined by

$$
\|\phi\|_{p ; \alpha, \beta ; n}:=\max _{j=0(1) n} I_{j}^{1 / p}(\phi) .
$$

As Kufner [2] has observed, weighted Sobolev spaces have applications in the theory of partial differential equations and in numerical methods for the solution of boundary-value problems. Elliott and Okada [1] have considered the finite Hilbert transform in the context of these spaces. In this paper we wish to consider the particular weakly

2010 AMS Mathematics subject classification. Primary 46E35, 47B37.

Received by the editors on February 13, 2014, and in revised form on June 2, 2014. 
singular operator which is denoted and defined by

$$
(A \phi)(t):=\int_{-1}^{1} \frac{\phi(\tau) d \tau}{|\tau-t| \gamma}, \quad \text { for } t \in \Omega \text { and } 0<\gamma<1,
$$

and its compactness on particular weighted Sobolev spaces.

For $1<p<\infty$, we define the conjugate number $q$ by

$$
\frac{1}{p}+\frac{1}{q}=1
$$

so that we always have $1<q<\infty$. In terms of this notation, Mikhlin and Prössdorf [3] have given the following theorem.

\section{Theorem 1.1. Suppose}

$$
1<p<\infty, \quad 0<\alpha+1 / q<1 \quad \text { and } \quad 0<\beta+1 / q<1 .
$$

Then $A$ is a compact operator on $W_{p ; \alpha, \beta}^{(0)}(\Omega)$ into itself.

Proof. See [3, Theorem 4.1, Chapter II].

We shall take this result as the starting point of our paper, and we shall prove, in Theorem 3.3, that Theorem 1.1 can be generalized to show that, under the conditions (5), $A$ is a compact operator on $W_{p ; \alpha, \beta}^{(n)}(\Omega)$ into itself, for all $n \in \mathbb{N}_{0}$.

Before embarking upon this, there are three immediate consequences of the definition of weighted Sobolev spaces which are worth noting. We shall let $D$ denote the differentiation operator and, furthermore, we shall define

$$
\rho(t):=1-t^{2} .
$$

Lemma 1.1. If $1 \leq p<\infty, \alpha$ and $\beta$ are real, and $n \in \mathbb{N}_{0}$, then

(i) $W_{p ; \alpha, \beta}^{(n)}(\Omega) \subset W_{p ; \alpha, \beta}^{(n-1)}(\Omega) \subset \cdots \subset W_{p ; \alpha, \beta}^{(0)}(\Omega)$;

(ii) $\phi \in W_{p ; \alpha, \beta}^{(n)}(\Omega)$ if and only if $\rho^{j} D^{j} \phi \in W_{p ; \alpha, \beta}^{(0)}(\Omega)$, for all $j=0(1) n$;

(iii) Under conditions (5), $A$ is a compact operator on $W_{p ; \alpha, \beta}^{(n)}(\Omega)$ into $W_{p ; \alpha, \beta}^{(0)}(\Omega)$ for all $n \in \mathbb{N}$. 
Proof. Parts (i) and (ii) are immediate consequences of Definition 1.1. The proof of part (iii) is an immediate consequence of Theorem 1.1 and part (i) of this lemma.

We shall need Hölder's inequalities for both integrals and sums. For $1<p<\infty$, these are given by

$$
\left|\int_{-1}^{1} \phi(\tau) \psi(\tau) d \tau\right| \leq\left(\int_{-1}^{1}|\phi(\tau)|^{p} d \tau\right)^{1 / p}\left(\int_{-1}^{1}|\psi(\tau)|^{q} d \tau\right)^{1 / q}
$$

and

$$
\left|\sum_{k=0}^{n} a_{k} b_{k}\right| \leq\left(\sum_{k=0}^{n}\left|a_{k}\right|^{p}\right)^{1 / p}\left(\sum_{k=0}^{n}\left|b_{k}\right|^{q}\right)^{1 / q}
$$

respectively, where $q$ is defined in (4). We will also need Leibnitz's theorem for the $n$th derivative of a product of two functions, which is given by

$$
D^{n}(\phi \psi)=\sum_{k=0}^{n}\left(\begin{array}{l}
n \\
k
\end{array}\right)\left(D^{k} \phi\right)\left(D^{n-k} \psi\right)
$$

for all $n \in \mathbb{N}$. Finally, throughout the paper, we will let $c$ denote a generic constant whose value may change from line to line. We may also write, for example, $c \neq c(a, b)$ in order to make the point that the constant $c$ is independent of $a$ and $b$, whatever these might happen to be.

In Section 2, under conditions (5) on $p, \alpha$ and $\beta$, we shall show the compactness of the operator $A$ on the space $W_{p ; \alpha, \beta}^{(1)}(\Omega)$ into itself and then, in Section 3, we will generalize this result to show the compactness of $A$ on $W_{p ; \alpha, \beta}^{(n)}(\Omega)$ into itself for all $n \in \mathbb{N}_{0}$.

2. The compactness of $A$ on the space $W_{p ; \alpha, \beta}^{(1)}(\Omega)$. We first need the following result.

Theorem 2.1. Suppose that $n \in \mathbb{N}, 1<p<\infty, 0<\alpha+1 / q<1$ and $0<\beta+1 / q<1$. Then $\phi \in W_{p ; \alpha, \beta}^{(n)}(\Omega)$ if and only if $\rho^{j} D^{j} \phi \in W_{p ; \alpha, \beta}^{(0)}(\Omega)$ for $j=1(1) n$. 
Proof. If $\phi \in W_{p ; \alpha, \beta}^{(n)}(\Omega)$, then, from Lemma 1.1 part (ii), we certainly have that $\rho^{j} D^{j} \phi \in W_{p ; \alpha, \beta}^{(0)}(\Omega)$ for $j=1(1) n$.

Suppose now that $\rho^{j} D^{j} \phi \in W_{p ; \alpha, \beta}^{(0)}(\Omega)$ for $j=1(1) n$. It then follows immediately from (1) that $I_{j}(\phi)<\infty$ for $j=1(1) n$. However, it remains to show that, if $\rho D \phi \in W_{p ; \alpha, \beta}^{(0)}(\Omega)$, then $\phi \in W_{p ; \alpha, \beta}^{(0)}(\Omega)$. We are given that $I_{1}(\phi)$ is finite, and we need to show that this implies the finiteness of $I_{0}(\phi)$. On defining

$$
I_{0,1}(\phi):=\int_{0}^{1}\left((1-\tau)^{-\alpha}(1+\tau)^{-\beta}|\phi(\tau)|\right)^{p} d \tau,
$$

we have, for $\tau \in[0,1)$,

$$
\begin{aligned}
\phi(\tau) & =\phi(0)+\int_{0}^{\tau} \phi^{\prime}(\xi) d \xi \\
& =\phi(0)+\int_{0}^{\tau}(1-\xi)^{\delta} \phi^{\prime}(\xi) \times(1-\xi)^{-\delta} d \xi,
\end{aligned}
$$

where $\delta$ is chosen so that

$$
1 / q<\delta<1-\alpha
$$

Recall from (5) that $1 / q<1-\alpha$ so that such a $\delta$ always exists. By Hölder's inequalities (7) and (8), it follows, from (11), that

$$
\leq c\left\{|\phi(0)|^{p}+\left(\int_{0}^{\tau}(1-\xi)^{p \delta}\left|\phi^{\prime}(\xi)\right|^{p} d \xi\right)\left(\int_{0}^{\tau}(1-\xi)^{-q \delta} d \xi\right)^{p / q}\right\},
$$

where $c \neq c(\tau, \phi)$. Since $1-q \delta<0$, we have

$$
\left(\int_{0}^{\tau}(1-\xi)^{-q \delta} d \xi\right)^{p / q} \leq c(1-\tau)^{p-1-p \delta},
$$

where $c \neq c(\tau)$. From (10), (13) and (14), we have

$$
\begin{aligned}
I_{0,1}(\phi) \leq & c\left\{|\phi(0)|^{p} \int_{0}^{1}(1-\tau)^{-p \alpha} d \tau\right. \\
& \left.+\int_{0}^{1}(1-\tau)^{-p \alpha-p \delta+p-1}\left(\int_{0}^{\tau}(1-\xi)^{p \delta}\left|\phi^{\prime}(\xi)\right|^{p} d \xi\right) d \tau\right\},
\end{aligned}
$$


where $c \neq c(\phi)$. On interchanging the order of integration in the iterated integral and observing, from (5), that $-p \alpha>-1$, we obtain

$$
I_{0,1}(\phi) \leq c\left\{|\phi(0)|^{p}+\int_{0}^{1}\left((1-\xi)^{1-\alpha}\left|\phi^{\prime}(\xi)\right|\right)^{p} d \xi\right\},
$$

where $c$ is independent of $\phi$. Since we are assuming that $\rho \phi^{\prime} \in$ $W_{p ; \alpha, \beta}^{(0)}(\Omega)$, it follows that the integral in (16) exists so that $I_{0,1}(\phi)$ is finite. By a similar argument over the interval $(-1,0]$, the details of which will not be given, it then follows that $I_{0}(\phi)$ is finite, so that the theorem is proved.

In order to continue with the proof of the compactness of $A$ on $W_{p ; \alpha, \beta}^{(1)}(\Omega)$ into itself, we need some further results.

Lemma 2.1. Suppose $\phi \in W_{p ; \alpha, \beta}^{(1)}(\Omega)$ with $1<p<\infty, 0<\alpha+1 / q<1$ and $0<\beta+1 / q<1$. Then $D(\rho \phi) \in W_{1 ; 0,0}^{(0)}(\Omega)$, together with

$$
(\rho \phi)(-1)=(\rho \phi)(+1)=0 \text {. }
$$

Proof. Since $\left|\rho^{\prime}(t)\right| \leq 2$ and $\rho(t) \geq 0$ for all $t \in \bar{\Omega}$, we have

$$
\begin{aligned}
\int_{-1}^{1}|D(\rho \phi)(\tau)| d \tau & \leq \int_{-1}^{1} 2|\phi(\tau)| d \tau+\int_{-1}^{1} \rho(\tau)\left|\phi^{\prime}(\tau)\right| d \tau \\
= & \int_{-1}^{1}\left((1-\tau)^{-\alpha}(1+\tau)^{-\beta}\left(2|\phi(\tau)|+\rho(\tau)\left|\phi^{\prime}(\tau)\right|\right)\right) \\
& \times(1-\tau)^{\alpha}(1+\tau)^{\beta} d \tau .
\end{aligned}
$$

Applying Hölder's inequalities, see (7) and (8), to this integral we find, since $\alpha+1 / q>0$ and $\beta+1 / q>0$, that

$$
\int_{-1}^{1}|D(\rho \phi)(\tau)| d \tau \leq c\|\phi\|_{p ; \alpha, \beta ; 1}<\infty
$$

where $c$ is independent of $\phi$. Since $\int_{-1}^{1}|D(\rho \phi)(\tau)| d \tau$ exists, we have that $D(\rho \phi)$ is integrable on $\Omega$, or that $D(\rho \phi) \in W_{1 ; 0,0}^{(0)}(\Omega)$, as required. 
To show that $\lim _{t \rightarrow 1} \rho(t) \phi(t)=0$, we have for $t \in[0,1)$,

$$
\begin{aligned}
\phi(t)= & \phi(0)+\int_{0}^{t}(1-\tau)^{1-\alpha}(1+\tau)^{1-\beta} \phi^{\prime}(\tau) \\
& \times(1-\tau)^{\alpha-1}(1+\tau)^{\beta-1} d \tau .
\end{aligned}
$$

By the triangle inequality and Hölder's inequality (7) we have

(21) $|\phi(t)| \leq|\phi(0)|+\|\phi\|_{p ; \alpha, \beta ; 1}\left(\int_{0}^{t}(1-\tau)^{q(\alpha-1)}(1+\tau)^{q(\beta-1)} d \tau\right)^{1 / q}$.

Since $t \in[0,1)$ and $q(1-\alpha)>1$, we find that

$$
\begin{aligned}
\int_{0}^{t}(1-\tau)^{q(\alpha-1)}(1+\tau)^{q(\beta-1)} d \tau & <2^{q(1-\beta)} \int_{0}^{t}(1-\tau)^{q(\alpha-1)} d \tau \\
& =\frac{c}{(1-t)^{q(1-\alpha)-1}}
\end{aligned}
$$

where $c=2^{q(1-\beta)} /(q(1-\alpha)-1)$. Inequalities (21) and (22) together give

$$
(1-t)|\phi(t)| \leq(1-t)|\phi(0)|+c\|\phi\|_{p ; \alpha, \beta ; 1}(1-t)^{\alpha+1 / q} .
$$

Since $\alpha+1 / q>0$, we see that $\lim _{t \rightarrow 1}(1-t)|\phi(t)|=0$. By arguing similarly at the end point -1 , we establish (17).

We shall now give conditions under which the operators $A$ and $D$ commute.

Theorem 2.2. Suppose $\phi \in W_{p ; \alpha, \beta}^{(1)}(\Omega)$ where

$$
1<p<\infty, \quad 0<\alpha+1 / q<1 \quad \text { and } \quad 0<\beta+1 / q<1 \text {. }
$$

Then, on $\Omega$,

$$
D A(\rho \phi)=A D(\rho \phi)
$$

Proof. Let us write $\psi=\rho \phi$. From (3), we have

$$
D A(\rho \phi)=\frac{d}{d t}\left\{\int_{-1}^{t} \frac{\psi(\tau)}{(t-\tau)^{\gamma}} d \tau+\int_{t}^{1} \frac{\psi(\tau)}{(\tau-t)^{\gamma}} d \tau\right\}
$$


Now

$$
\int_{-1}^{t} \frac{\psi(\tau)}{(t-\tau)^{\gamma}} d \tau=-\frac{1}{1-\gamma} \int_{-1}^{t} \psi(\tau) d\left((t-\tau)^{1-\gamma}\right)
$$

On integrating by parts and recalling, from Lemma 2.1 , that $\psi(-1)=0$, we find, since $1-\gamma>0$,

$$
\int_{-1}^{t} \frac{\psi(\tau)}{(t-\tau)^{\gamma}} d \tau=\frac{1}{1-\gamma} \int_{-1}^{t}(t-\tau)^{1-\gamma}(D \psi)(\tau) d \tau
$$

On differentiating with respect to $t$, see for example Olver et al. [4, equation (1.5.22)], we have

$$
\frac{d}{d t} \int_{-1}^{t} \frac{\psi(\tau)}{(t-\tau)^{\gamma}} d \tau=\int_{-1}^{t} \frac{D(\rho \phi)(\tau)}{(t-\tau)^{\gamma}} d \tau .
$$

Arguing similarly, we find that

$$
\frac{d}{d t} \int_{t}^{1} \frac{\psi(\tau)}{(\tau-t)^{\gamma}} d \tau=\int_{t}^{1} \frac{D(\rho \phi)(\tau)}{(\tau-t)^{\gamma}} d \tau .
$$

From (28) and (29), we obtain (24), as required.

At this point it is convenient to introduce two further linear operators.

Definition 2.1. For $\phi \in W_{p ; \alpha, \beta}^{(0)}(\Omega)$, we define, on $\Omega$,

$$
B \phi:=A(\rho \phi)-\rho A \phi \quad \text { and } \quad C \phi:=A\left(\rho^{\prime} \phi\right)-\rho^{\prime} A \phi .
$$

Theorem 2.3. For $\phi \in W_{p ; \alpha, \beta}^{(1)}(\Omega)$ with $1<p<\infty, 0<\alpha+1 / q<1$ and $0<\beta+1 / q<1$ we have, for $t \in \Omega$,

$$
D B \phi(t)=(2-\gamma) t(A \phi)(t)-\gamma A(t \phi)(t)
$$

and

$$
C \phi(t)=2 t(A \phi)(t)-2 A(t \phi)(t)
$$

so that the operators $D B$ and $C$ are compact operators on $W_{p ; \alpha, \beta}^{(1)}(\Omega)$ into $W_{p ; \alpha, \beta}^{(0)}(\Omega)$. 
Proof. From (3), (6) and (30) we have, for $t \in \Omega$,

$$
\begin{aligned}
D B \phi(t)= & \frac{d}{d t}\left\{\int_{-1}^{1} \frac{(t-\tau)(t+\tau)}{|\tau-t|^{\gamma}} \phi(\tau) d \tau\right\} \\
= & \frac{d}{d t}\left\{\int_{-1}^{t}(t-\tau)^{1-\gamma}(t+\tau) \phi(\tau) d \tau\right. \\
& \left.-\int_{t}^{1}(\tau-t)^{1-\gamma}(t+\tau) \phi(\tau) d \tau\right\} .
\end{aligned}
$$

Since $1-\gamma>0$, we find on performing the differentiation that

$$
\begin{aligned}
D B \phi(t) & =(1-\gamma) \int_{-1}^{1} \frac{(t+\tau)}{|\tau-t| \gamma} \phi(\tau) d \tau+\int_{-1}^{1} \frac{(t-\tau)}{|\tau-t|^{\gamma}} \phi(\tau) d \tau \\
& =(1-\gamma)(t(A \phi)(t)+A(t \phi)(t))+t(A \phi)(t)-A(t \phi)(t)
\end{aligned}
$$

from which (31) follows at once.

From (6) and (30), (32) follows immediately.

Since $\phi \in W_{p ; \alpha, \beta}^{(1)}(\Omega)$, we have that both $\phi$ and $t \phi$ are in $W_{p ; \alpha, \beta}^{(0)}(\Omega)$. It then follows, from Theorem 1.1, that the operators $D B$ and $C$ are compact on $W_{p ; \alpha, \beta}^{(1)}(\Omega)$ into $W_{p ; \alpha, \beta}^{(0)}(\Omega)$, each being the sum of two compact operators.

We need to define here one further operator which, together with its generalization in Section 3, will be of considerable importance in this analysis.

Definition 2.2. For $\phi \in W_{p ; \alpha, \beta}^{(1)}(\Omega)$, we define, on $\Omega$, the operator $\Delta_{1}$ by

$$
\Delta_{1} \phi:=A(\rho D \phi)-\rho D A \phi
$$

We are now in a position to relate the operator $\Delta_{1}$ to operators $B$, $C$ and $D$.

Theorem 2.4. Suppose $\phi \in W_{p ; \alpha, \beta}^{(1)}(\Omega)$ with

$$
1<p<\infty, \quad 0<\alpha+1 / q<1 \text { and } 0<\beta+1 / q<1 .
$$


Then, on $\Omega$,

$$
\Delta_{1} \phi=D B \phi-C \phi
$$

so that $\Delta_{1}$ is a compact operator on $W_{p ; \alpha, \beta}^{(1)}(\Omega)$ into $W_{p ; \alpha, \beta}^{(0)}(\Omega)$.

Proof. From (30), we have, on $\Omega$,

$$
D B \phi-C \phi=D A(\rho \phi)-\rho D A \phi-A\left(\rho^{\prime} \phi\right) .
$$

From (24), it now follows at once that

$$
D B \phi-C \phi=A(\rho D \phi)-\rho D A \phi=\Delta_{1} \phi,
$$

from (35), as claimed. Since, from Theorem 2.3 both $D B$ and $C$ are compact operators on $W_{p ; \alpha, \beta}^{(1)}(\Omega)$ into $W_{p ; \alpha, \beta}^{(0)}(\Omega)$, then so is $\Delta_{1}$, it being the sum of these two compact operators.

It now remains to show that the operator $A$ is a compact operator on $W_{p ; \alpha, \beta}^{(1)}(\Omega)$ into itself, under the usual conditions.

Theorem 2.5. Suppose

$$
1<p<\infty, \quad 0<\alpha+1 / q<1 \quad \text { and } \quad 0<\beta+1 / q<1 .
$$

Then $A$ is a compact operator on $W_{p ; \alpha, \beta}^{(1)}(\Omega)$ into itself.

Proof. Suppose $\phi \in W_{p ; \alpha, \beta}^{(1)}(\Omega)$. Then, from Lemma 1.1 (i), we also have that $\phi \in W_{p ; \alpha, \beta}^{(0)}(\Omega)$ so that, from Theorem 1.1, it follows that $A$ is a compact operator on $W_{p ; \alpha, \beta}^{(1)}(\Omega)$ into $W_{p ; \alpha, \beta}^{(0)}(\Omega)$.

Again, since from Lemma 1.1 (ii) we have that $\rho D \phi \in W_{p ; \alpha, \beta}^{(0)}(\Omega)$ it follows, from Theorem 1.1 again, that $A(\rho D)$ is a compact operator on $W_{p ; \alpha, \beta}^{(1)}(\Omega)$ into $W_{p ; \alpha, \beta}^{(0)}(\Omega)$. Since, from $(38)$, we have that $\rho D(A \phi)=$ $A(\rho D \phi)-\Delta_{1} \phi$ and since, in Theorem 2.4, we have shown that $\Delta_{1}$ is a compact operator on $W_{p ; \alpha, \beta}^{(1)}(\Omega)$ into $W_{p ; \alpha, \beta}^{(0)}(\Omega)$, it follows that $\rho D A$ is a compact operator on $W_{p ; \alpha, \beta}^{(1)}(\Omega)$ into $W_{p ; \alpha, \beta}^{(0)}(\Omega)$.

Given any sequence of functions $\left\{\phi_{m}\right\}_{m \in \mathbb{N}}$, where $\phi_{m} \in W_{p ; \alpha, \beta}^{(1)}(\Omega)$, it follows that there exists a subsequence $\left\{\phi_{1, m}\right\}$ say, such that the 
sequence $\left\{A \phi_{1, m}\right\}$ converges to $\psi_{0}$ and the sequence $\left\{\rho D A \phi_{1, m}\right\}$ converges to $\psi_{1}$, where the functions $\psi_{0}$ and $\psi_{1}$ are in $W_{p ; \alpha, \beta}^{(0)}(\Omega)$. Consequently, $\rho D \psi_{0}=\psi_{1}$ so that, since $\psi_{1} \in W_{p ; \alpha, \beta}^{(0)}(\Omega)$, it follows from Theorem 2.1 that $\psi_{0} \in W_{p ; \alpha, \beta}^{(1)}(\Omega)$. Since $\left\{\phi_{m}\right\}$ was any sequence of functions in $W_{p ; \alpha, \beta}^{(1)}(\Omega)$ and since the sequence $\left\{A \phi_{1, m}\right\}$ converges to an element of $W_{p ; \alpha, \beta}^{(1)}(\Omega)$, we have that $A$ is a compact operator on $W_{p ; \alpha, \beta}^{(1)}(\Omega)$ into itself.

3. The compactness of $A$ on $W_{p ; \alpha, \beta}^{(n)}(\Omega)$ into itself for all $n \in \mathbb{N}_{0}$. First, let us generalize Definition 2.2.

Definition 3.1. For all $n \in \mathbb{N}$ and $\phi \in W_{p ; \alpha, \beta}^{(n)}(\Omega)$, we define on $\Omega$ the operator $\Delta_{n}$ by

$$
\Delta_{n} \phi:=A\left(\rho^{n} D^{n} \phi\right)-\rho^{n} D^{n} A \phi .
$$

Theorem 3.1. Suppose $n \in \mathbb{N}$ with

$$
1<p<\infty, \quad 0<\alpha+1 / q<1 \text { and } 0<\beta+1 / q<1 .
$$

Assume that, for $j=1(1) n, \Delta_{j}$ is a compact operator on $W_{p ; \alpha, \beta}^{(j)}(\Omega)$ into $W_{p ; \alpha, \beta}^{(0)}(\Omega)$. Then $A$ is a compact operator on $W_{p ; \alpha, \beta}^{(n)}(\Omega)$ into itself.

Proof. We shall first show that, under the given conditions, $A$ maps the space $W_{p ; \alpha, \beta}^{(n)}(\Omega)$ into itself. Suppose $\phi \in W_{p ; \alpha, \beta}^{(n)}(\Omega)$. Then since, from Lemma 1.1 (ii), $\rho^{j} D^{j} \phi \in W_{p ; \alpha, \beta}^{(0)}(\Omega)$ for $j=1(1) n$, it follows from Theorem 1.1 that $A\left(\rho^{j} D^{j} \phi\right)$ is also in $W_{p ; \alpha, \beta}^{(0)}(\Omega)$, for $j=1(1) n$. From (39), we have that $\rho^{j} D^{j} A \phi=A\left(\rho^{j} D^{j} \phi\right)-\Delta_{j} \phi$, for $j=1(1) n$. Since we are assuming that $\Delta_{j}$ maps $W_{p ; \alpha, \beta}^{(j)}(\Omega)$ into $W_{p ; \alpha, \beta}^{(0)}(\Omega)$ for $j=1(1) n$, it then follows that $\rho^{j} D^{j} A$ maps $W_{p ; \alpha, \beta}^{(j)}(\Omega)$ into $W_{p ; \alpha, \beta}^{(0)}(\Omega)$ for all $j=1(1) n$. As a consequence of Lemma 1.1 (ii) again, we have that $A \phi \in W_{p ; \alpha, \beta}^{(n)}(\Omega)$ or, in other words, $A$ maps $W_{p ; \alpha, \beta}^{(n)}(\Omega)$ into itself.

To show the compactness of $A$, we can argue as we have done in the proof of Theorem 2.5. Let $\left\{\phi_{m}\right\}, m \in \mathbb{N}$, be any bounded sequence of functions in $W_{p ; \alpha, \beta}^{(n)}(\Omega)$. Then we can ultimately find a 
subsequence of functions $\left\{\phi_{1, m}\right\}, m \in \mathbb{N}$, such that, for each $j=1(1) n$, the sequence $\left\{\rho^{j} D^{j}\left(A \phi_{1, m}\right)\right\}$ converges to a function $\psi_{j}$, say, where each $\psi_{j} \in W_{p ; \alpha, \beta}^{(0)}$. It then follows, from Theorem 2.1, that the limit of the sequence $\left\{A \phi_{1, m}\right\}$ is an element of $W_{p ; \alpha, \beta}^{(n)}(\Omega)$. That is, $A$ is a compact operator on $W_{p ; \alpha, \beta}^{(n)}(\Omega)$ into itself.

On recalling Definition 2.1 for the operators $B$ and $C$, we can now prove the following important result.

Theorem 3.2. For all $n \in \mathbb{N}$ and $\phi \in W_{p ; \alpha, \beta}^{(n+1)}(\Omega)$, we have that

$$
\begin{aligned}
\Delta_{n+1} \phi & =\Delta_{n}(\rho D \phi)-n \rho^{\prime} \Delta_{n} \phi+n(n-1) \rho \Delta_{n-1} \phi \\
& +\rho^{n} D^{n} \Delta_{1} \phi-n C\left(\rho^{n} D^{n} \phi\right)+n(n-1) B\left(\rho^{n-1} D^{n-1} \phi\right) .
\end{aligned}
$$

Proof. From equation (39), we have, for all $n \in \mathbb{N}$,

$$
\Delta_{n}(\rho D \phi)=A\left(\rho^{n} D^{n}(\rho D \phi)\right)-\rho^{n} D^{n} A(\rho D \phi) .
$$

Recalling, from $(6)$, that $\rho(t)=1-t^{2}$, we have by Leibnitz's theorem, see $(9)$, that

$$
D^{n}(\rho D \phi)=\rho D^{n+1} \phi+n \rho^{\prime} D^{n} \phi-n(n-1) D^{n-1} \phi .
$$

Since, from (35), $A(\rho D \phi)=\Delta_{1} \phi+\rho D A \phi$, it follows, from (41) and (42), that

$$
\begin{array}{r}
\Delta_{n}(\rho D \phi)=A\left(\rho^{n+1} D^{n+1} \phi\right)-\rho^{n} D^{n} \Delta_{1} \phi-\rho^{n} D^{n}(\rho D A \phi) \\
+n A\left(\rho^{\prime} \rho^{n} D^{n} \phi\right)-n(n-1) A\left(\rho \rho^{n-1} D^{n-1} \phi\right) .
\end{array}
$$

But again, from (9),

$$
\rho^{n} D^{n}(\rho D A \phi)=\rho^{n+1} D^{n+1} A \phi+n \rho^{\prime} \rho^{n} D^{n} A \phi-n(n-1) \rho^{n} D^{n-1} A \phi .
$$

On substituting (44) into (43) and recalling the definition of $\Delta_{n+1} \phi$ from (39), we obtain

$$
\begin{array}{r}
\Delta_{n+1} \phi=\Delta_{n}(\rho D \phi)+\rho^{n} D^{n} \Delta_{1} \phi+n\left(\rho^{\prime} \rho^{n} D^{n} A \phi-A\left(\rho^{\prime} \rho^{n} D^{n} \phi\right)\right) \\
+n(n-1)\left(A\left(\rho \rho^{n-1} D^{n-1} \phi\right)-\rho . \rho^{n-1} D^{n-1} A \phi\right) .
\end{array}
$$


From equations (30) and (39), this gives

$$
\begin{aligned}
& \Delta_{n+1} \phi=\Delta_{n}(\rho D \phi)+\rho^{n} D^{n} \Delta_{1} \phi-n C\left(\rho^{n} D^{n} \phi\right)-n \rho^{\prime} \Delta_{n} \phi \\
& +n(n-1)\left(B\left(\rho^{n-1} D^{n-1} \phi\right)+\rho\left(A\left(\rho^{n-1} D^{n-1} \phi\right)-\rho^{n-1} D^{n-1} A \phi\right)\right) .
\end{aligned}
$$

On recalling the definition of $\Delta_{n-1} \phi$ from (39) we see that (40) follows.

We now come to the principal result of this paper.

Theorem 3.3. Suppose $n \in \mathbb{N}_{0}$ with

$$
1<p<\infty, \quad 0<\alpha+1 / q<1 \quad \text { and } \quad 0<\beta+1 / q<1 .
$$

Then $A$ is a compact operator on $W_{p ; \alpha, \beta}^{(n)}(\Omega)$ into itself.

Proof. We shall prove this by mathematical induction. Recall that the theorem is true when $n=0$ (Theorem 1.1) and when $n=1$ (Theorem 2.5) so that we need to show that it is true for all $n \geq 2$. In Theorem 3.1, on assuming that for $j=1(1) n, \Delta_{j}$ mapped $W_{p ; \alpha, \beta}^{(j)}(\Omega)$ compactly into $W_{p ; \alpha, \beta}^{(0)}(\Omega)$, it followed that $A$ mapped $W_{p ; \alpha, \beta}^{(n)}(\Omega)$ compactly into itself. The theorem will therefore follow if we can show that $\Delta_{n+1}$ maps $W_{p ; \alpha, \beta}^{(n+1)}(\Omega)$ compactly into $W_{p ; \alpha, \beta}^{(0)}(\Omega)$. Let us consider each term of (40).

For $\phi \in W_{p ; \alpha, \beta}^{(n+1)}(\Omega)$, we have, from Lemma 1.1 (i), that both $\phi$ and $\rho D \phi$ are in $W_{p ; \alpha, \beta}^{(n)}(\Omega)$. Consequently, the operator

$$
\Delta_{n}(\rho D)-n \rho^{\prime} \Delta_{n}+n(n-1) \rho \Delta_{n-1}
$$

maps $W_{p ; \alpha, \beta}^{(n+1)}(\Omega)$ compactly into $W_{p ; \alpha, \beta}^{(0)}(\Omega)$. For the terms in $(40)$ involving the operators $B$ and $C$ we have from Lemma 1.1 (ii) that both $\rho^{n-1} D^{n-1} \phi$ and $\rho^{n} D^{n} \phi$ are in $W_{p ; \alpha, \beta}^{(0)}(\Omega)$. It then follows from (30) and Theorem 1.1 that both $B\left(\rho^{n-1} D^{n-1}\right)$ and $C\left(\rho^{n} D^{n}\right)$ are compact operators on $W_{p ; \alpha, \beta}^{(n+1)}(\Omega)$ into $W_{p ; \alpha, \beta}^{(0)}(\Omega)$. It now remains to consider the term $\rho^{n} D^{n} \Delta_{1} \phi$.

From equations (6), (30), (31) and (36) it follows that

$$
\Delta_{1} \phi=(2-\gamma) A(t \phi)-\gamma t A \phi
$$


Since we are assuming that $A$ is a compact operator on $W_{p ; \alpha, \beta}^{(n)}(\Omega)$ into itself it follows, from (47), that $\Delta_{1}$ is also a compact operator on $W_{p ; \alpha, \beta}^{(n)}(\Omega)$ into itself. Consequently, from Lemma 1.1 (ii), we have that $\rho^{n} D^{n} \Delta_{1}$ is a compact operator on $W_{p ; \alpha, \beta}^{(n)}(\Omega)$ into $W_{p ; \alpha, \beta}^{(0)}(\Omega)$ and, therefore, by Lemma $1.1(\mathrm{i})$, on $W_{p ; \alpha, \beta}^{(n+1)}(\Omega)$ into $W_{p ; \alpha, \beta}^{(0)}(\Omega)$.

Putting these results together we see, from (40), that $\Delta_{n+1}$ is a compact operator on $W_{p ; \alpha, \beta}^{(n+1)}(\Omega)$ into $W_{p ; \alpha, \beta}^{(0)}(\Omega)$ so that, from Theorem 3.1, it follows that $A$ is a compact operator on $W_{p ; \alpha, \beta}^{(n+1)}(\Omega)$ into itself. The theorem now follows immediately by induction.

4. Final remarks. Although we shall not prove it here, it turns out that Theorem 1.1 is also true when $p=1,0<\alpha<1$ and $0<\beta<1$. Under these same conditions, Theorem 3.3 is also true.

We have considered only one particular weakly singular operator. Another important one is $L$ where, for $t \in \Omega$, we define

$$
L \phi(t):=\int_{-1}^{1} \log |\tau-t| \phi(\tau) d \tau .
$$

From Mikhlin and Prössdorf [3] it follows that Theorem 1.1 is also true for the operator $L$. By doing an analysis similar to that in Sections 2 and 3 , it can be shown that Theorem 3.3 is also true for the operator $L$, but we shall not give the details here.

Acknowledgments. The author is extremely grateful to his colleague Dr. Susumu Okada for all the help and assistance he has given in the production of this paper.

\section{REFERENCES}

1. David Elliott and Susumu Okada, The finite Hilbert transform and weighted Sobolev spaces, Math. Nachr. 266 (2004), 34-47.

2. A. Kufner, Weighted Sobolev spaces, Wiley \& Sons, New York, 1985.

3. S.G. Mikhlin and S. Prössdorf, Singular integral operators, Springer-Verlag, Berlin, 1986.

4. F.W.J. Olver, et al., NIST Handbook of mathematical functions, NIST and Cambridge University Press, New York, 2010. 
School of Mathematics and Physics, University of Tasmania, Private Bag 37, Hobart, Tasmania 7001, Australia

Email address: David.Elliott@utas.edu.au 